\title{
H-plane SIW active horn antenna
}

\author{
N. Esparza, P. Alcón, L.F. Herrán, F. Las-Heras \\ Signal and Communications Theory Area, Department Electrical Engineering, University of Oviedo \\ E-33203 Gijón, Spain. \\ \{nesparza, palcon, lfherran, flasheras\}@tsc.uniovi.es
}

\begin{abstract}
This paper presents a H-plane active horn antenna based on SIW technology. The amplifiers are fully integrated within the SIW structure by replacing the top layer by a frequency selective surface operating in stop-band. Results show 3 to $5 \mathrm{~dB}$ gain improvement in the $\mathrm{H}$-plane and $\mathrm{E}$-plane measurements.
\end{abstract}

Keywords-SIW, horn antenna, amplifiers, active antenna.

\section{INTRODUCTION}

Substrate integrated waveguide (SIW) technology is having high development since it was presented in early 2000s. This interest is based on the fact of the high versatility and performance achieved by this technology while implementing passive elements such as filters, couplers, antennas, etc., at a reduced size and cost. Up to now, much of the designing efforts have focused in passive elements and how to realize multidimensional techniques for high density integration [1]. However, few experiments have been done in order to integrate amplifiers in SIW waveguides. In these experiments, the amplifier is not fully integrated within the SIW or they use different SIW topologies [2][3]. Some efforts have also been done to integrate active elements with SIW patch antennas [4]. However, to our knowledge no active SIW horn has been presented.

In this paper, we present a novel H-plane SIW horn antenna with the amplifiers fully integrated within the SIW structure by means of replacing the top conducting layer by a frequency selective surface operating in its stop-band. This inclusion assures DC isolation making the SIW antenna fully compatible with amplifier biasing.

\section{ANTENNA TOPOLOGY}

The antenna is designed as a X-band 3-layer H-plane SIW active horn. Each layer is designed in a $1.524 \mathrm{~mm}$ thickness RO4003C substrate. In order to minimize the layer bending, some screws are added in the horn antenna. Screws insertion is also used to obtain an extra phase correction, following the rules in [5]. The metallic top side of the antenna is replaced by a stopband FSS. If the FSS is properly designed, it doesn't radiate and it behaves as a perfect conductor plate as it is shown in [6], allowing DC isolation and thus making this surface compatible with amplifiers integration.

In order to get the maximum energy to be applied into the amplifier, a taper is implemented. By doing this, the structure concentrates the energy flowing around the taper location, so that the amplifier can take it. The amount of energy condensed by the taper will greatly depends on its position and the propagating mode in the SIW. For example if the tapers are placed near the symmetry axis of the antenna and a dominant

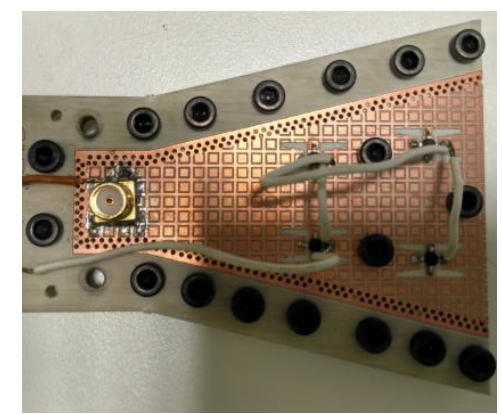

Fig.1. Implemented active antenna photograph.

$T E_{10}$ mode propagates, the taper will take more energy that in the case in which the tapers are placed near the SIW walls. For that reason, the gain improvement of the antenna could be different from the maximum gain of the amplifier as a result of the taper position. However, the introduction of amplifiers in some areas of the antenna could be exploited to modify the radiation pattern of the antenna, as a consequence of a local field amplification and a phase variation. As in every topology where several amplifiers are connected in parallel, all of them should exhibit the most similar performance to ensure symmetrical behavior if desired. The proposed structure, shown in Fig. 1, is a four amplifiers structure divided into two levels and its main aim is show the availability of $\mathrm{H}$-plane SIW active horn antennas. The amplifiers are not placed in the maximum energy area so the expected gain improvement will be less than the maximum available gain of the amplifiers.

\section{EXPERIMENTAL RESULTS}

The return losses of the complete active antenna are shown in Fig. 2. They are plotted within the range where the radiation patterns are measured. Return losses are not much different

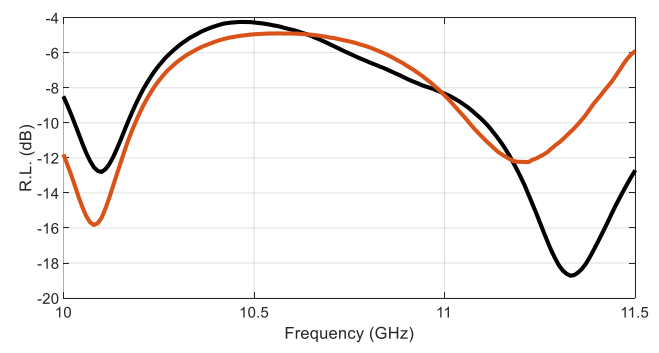

Fig. 2. Active antenna return losses. Black line: unbiased antenna. Red line: biased antenna 


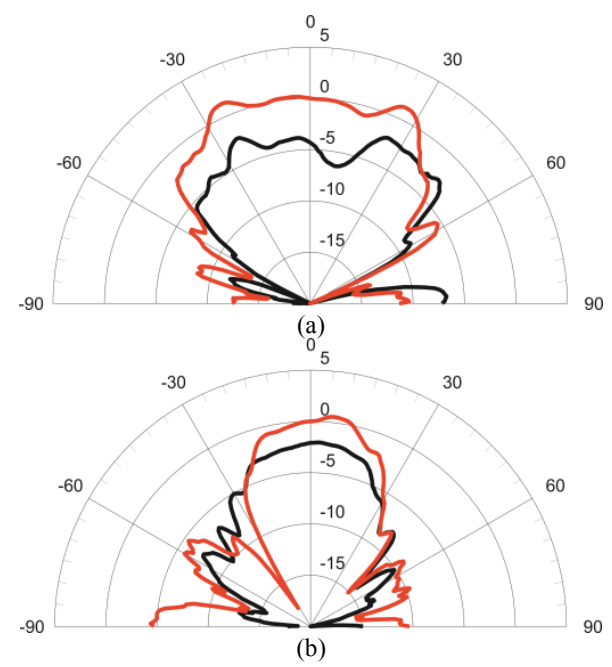

Fig.3. H-plane antenna radiation pattern. Red line: biased amplifiers. Black line: unbiased amplifiers. (a) $10.25 \mathrm{GHz}$. (b) $11.5 \mathrm{GHz}$.

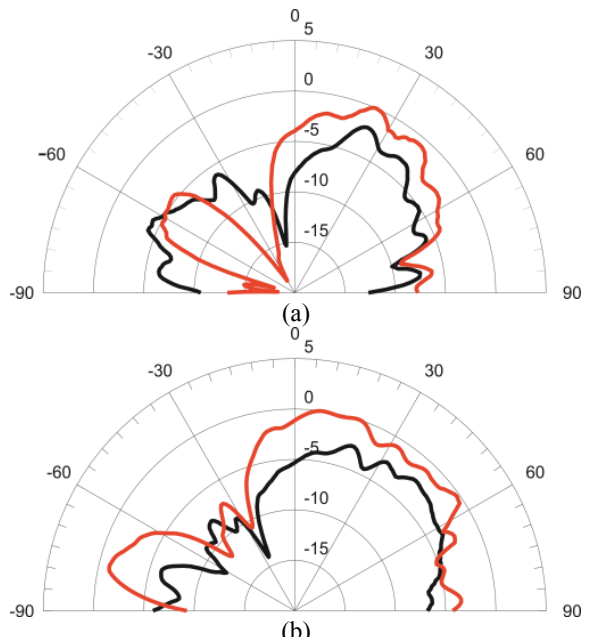

(b)

Fig.4. E-plane antenna radiation pattern. Red line: biased amplifiers. Black line: unbiased amplifiers. (a) $10.25 \mathrm{GHz}$. (b) $11.5 \mathrm{GHz}$.

from the SIW horn antenna without amplifiers. If better return losses are desired, techniques presented in [7] can be used. In order to show how the radiation pattern of the antenna is affected by the amplifiers, the H-plane and E-plane radiation patterns are measured at two different frequencies, where good return losses are achieved, and in two different bias conditions. First measure is done letting the amplifiers unbiased and it is compared with the same measurement setup but letting the amplifiers biased. These measurements are shown in Figs. 3 and 4. The radiation patterns are normalized to the maximum value of the antenna with biased amplifiers in broadside direction for $\mathrm{H}$-plane and to the maximum value with biased amplifiers for E-plane. As it can be seen, the radiation pattern shape is not the typical that could be obtained by a SIW horn. This is due to three main factors. The first one is the use of screws that corrects the phase. The second one is some misalignment between layers. The third one is the use of amplifiers which are changing the field inside the horn. Depending on the considered frequency, there is a gain improvement from 3 to $5 \mathrm{~dB}$ while the amplifiers are biased. As explained in section II, the amplifiers are not placed in the maximum energy area, so they are taking only a limited percentage of the energy flowing through the antenna producing a decrease in total gain that could be achieved. In this particular case, the maximum available gain could be up to $8 \mathrm{~dB}$. However, the results show how it is possible to create SIW active horn antennas and how the total gain improvement will depend on the amplifiers positions.

\section{CONCLUSIONS}

A multilayer SIW active horn antenna is presented. In order to add amplifiers to the upper side of the antenna, such side is replaced by a FSS working in its stop-band, allowing DC isolation and bias compatibility. Results show that the addition of amplifiers in the structure maintain its return losses almost unaffected compared with its counterpart passive SIW horn antenna. In addition, the proposed configuration increases the total gain of the antenna. Gain improvement will depend on the number of amplifiers and its position in the SIW horn antenna. Results show the availability of a H-plane SIW active horn antenna.

\section{ACKNOWLEDGMENT}

This work was supported by the Gobierno del Principado de Asturias under the Plan de Ciencia Tecnología e Innovación (PCTI) by grants BP13042 and BP12032, by the Gobierno del Principado de Asturias and FEDER (Unión Europea) under project GRUPIN14-114, and the Ministerio de Economía y Competitividad under project TEC2014-54005-P.

\section{REFERENCES}

[1] Ali Doghri et al., "Multi-dimensional substrate integrated waveguide for high density integration”, 2015 Global Symposium on Millimeter waves (GSMM), May 2015.

[2] M. Abdolhamidi et al., "X-Band substrate integrated waveguide amplifier", IEEE microwave and wireless components letters, vol. 18, no12, December 2008.

[3] K.W. Eccleston, "Corrugated Substrate Integrated Waveguide distributed Amplifier", Asia Pacific Microwaves conference, 2012.

[4] F. Giuppi et al., "Active substrate integrated waveguide (SIW) antenna with phase-shifterless beam-scanning capabilities", International IEEE MTT-S, 2012.

[5] Wang L. et al., "Phase corrected substrate integrated waveguide H-plane horn antenna with embebbed metal-via arrays", IEEE trans. on antennas and propagation vol. 62, pp.1854-1861, 2014.

[6] N. Esparza et al., "Substrate Integrated Waveguides structures using frequency selective surfaces operating in stop-band (SBFSS-SIW)" IEEE microwave an wireless components letters, vol. 26, no.2, 2016.

[7] M.Esquius-Morote et al., "A printed transition for matching improvement of SIW Horn Antennas”, IEEE Trans. Antennas Propag., vol. 61, no.4, pp. 1923-1930, April 2013 